\title{
Simulation of Decoherence in Fluctuation Electron Microscopy
}

\author{
A. Rezikyan ${ }^{1}$, Z. Jibben ${ }^{2}$, B. Rock ${ }^{1}$, G. Zhao ${ }^{3}$ and M. M. J. Treacy ${ }^{1}$ \\ ${ }^{1}$ Dept. of Physics, Arizona State University, Tempe, AZ 85287 USA \\ 2 Ira A. Fulton School of Engineering, Arizona State University, Tempe, AZ 85287 USA \\ ${ }^{3}$ Dept. of Structural Biology, University of Pittsburgh School of Medicine, Pittsburgh, PA 15260 \\ USA
}

Fluctuation Electron Microscopy (FEM) examines the scattering statistics from small volumes of thin amorphous materials to detect the presence of medium-range order (MRO) [1]. By now it has been thoroughly demonstrated, by both modeling and simulations, that FEM is extraordinarily sensitive to MRO, much more than high-resolution diffraction and high-resolution imaging. Experiments have confirmed this sensitivity [2-4]. There are two key contributing factors to this sensitivity. First, FEM examines the variance of the scattering statistics, which is the second moment of the intensity distribution. Essentially, FEM examines the speckliness of the diffraction data. This means that it examines four-body correlations, whereas diffraction alone examines the first moment of intensity, and examines pair-correlations. The four-body terms are much more sensitive to medium-range correlations. The second key factor, which in this era of aberration-corrected microscopy may come as a surprise, is that it is a low-resolution technique. The sensitivity of FEM to MRO is maximized when the resolution is comparable to the length scale of the MRO.

Although FEM is successful as a qualitative technique - it can disclose unambiguously and sensitively the signature of MRO in a sample - it is not yet truly quantitative. There are two main reasons for this state of affairs. We do not know how to invert analytically four-body diffraction data. There has been significant progress in bypassing this issue by use of the experimentally constrained reverse Monte Carlo method [5-7]. This method shows great promise, but it has revealed a huge discrepancy between simulated variance and experimental variance: the experimental variance is usually a factor of 10-100 less than the calculated values and it is suppressed much more severely at scattering vector values exceeding $10 \mathrm{~nm}^{-1}$.

This discrepancy between experimental variance and computed variance was attributed to the illumination incoherence in early FEM studies. A model of such incoherence was developed [8], where it was assumed that if there were $m$ incoherent sources, then the intensity probability distribution in a tilted dark-field image would be

$$
I^{\prime}(l)-\frac{m^{m}}{(m-1) !} \frac{l^{m} \text { । }}{\bar{l}^{m}} \exp \left(-\frac{m l}{\bar{l}}\right) .
$$

where $I$ is the mean intensity. This formula is obeyed remarkably well for data obtained from tilted dark-field TEM images of amorphous carbon (Figure 1 (a) and (b)). Fits to that data give $m \approx 30$, yet we know experimentally that the illumination is highly coherent, suggesting that we should be nearer $m \approx$ 1. This result indicates that we have been misinterpreting the meaning of $m$ in this formula.

This surprise result points us towards an alternative source for $m$-decoherence of the scattering within the sample. In essence, this is a type of diffuse scattering, but it appears to be induced mainly by beam knock-on damage in the sample, which subtly alters the underlying structure being probed during the 
exposure of the diffraction patterns. In turn, this causes the speckle intensity to fade when speckle is time-averaged over the several seconds needed to record the data. Multiple scattering may be another source of decoherence. Ironically, because FEM is so sensitive to such fluctuations, it is therefore much strongly affected by decoherence than are simple diffraction and imaging.

Several heuristic models of decoherence in FEM were simulated to explain qualitatively the differences between calculations and experiment. It appeared that the combination of the knock-on damage and the multiple scattering models result in suppression of the whole plot of the normalized variance as well as a much severe suppression at high scattering vector, $\kappa$ (Figure $1(\mathrm{c})$ ). The baseline normalized-variance is equal to 1 for the fully coherent kinematical case of the red plot, as it is 1 for the speckle pattern of a completely random structure (the peaks arise because the model structure was not completely random, but rather a polycrystalline silicon). A pure statistical result suggests $1 / \mathrm{m}$ scaling of the normalized variance for the speckle pattern, which is a combination of $m$ random kinematical speckle patterns. Therefore, the blue plot effectively puts in decoherence of $m \approx 16$ since its baseline is suppressed by the factor of 16 as it is seen from Figure 1 (c).

\section{References}

[1] M.M.J. Treacy et al., Rep. Prog. Phys., 2005. 68: p. 2899-2944.

[2] J.M. Gibson and M.M.J. Treacy,. Phys. Rev. Lett., 1997. 78: p. 1074-1077.

[3] P.M. Voyles and J.R. Abelson, Solar Energy Materials and Solar Cells, 2003. 78: p. 85-113.

[4] M.M.J. Treacy et al., J. Non-Cryst. Solids, 1998. 231: p. 99-110.

[5] P. Biswas et al., Phys. Rev. B, 2004. 69: p. 195207.

[б] K.B. Borisenko et al., Submitted to Acta Materiala, 2011.

[7] J. Hwang et al., Journal of Materials Research, 2009. 24: p. 3121-3129.

[8] M.M.J. Treacy and J.M. Gibson,. Inst. Phys. Conf. Ser. No 153, 1997: p. 433-436.

[9] Authors gratefully acknowledge the DOE for funding under contract number DE-SC0004929.

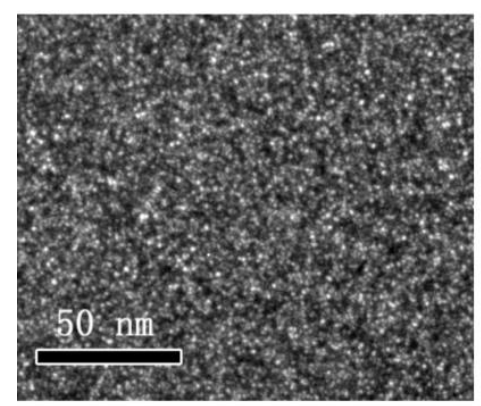

a
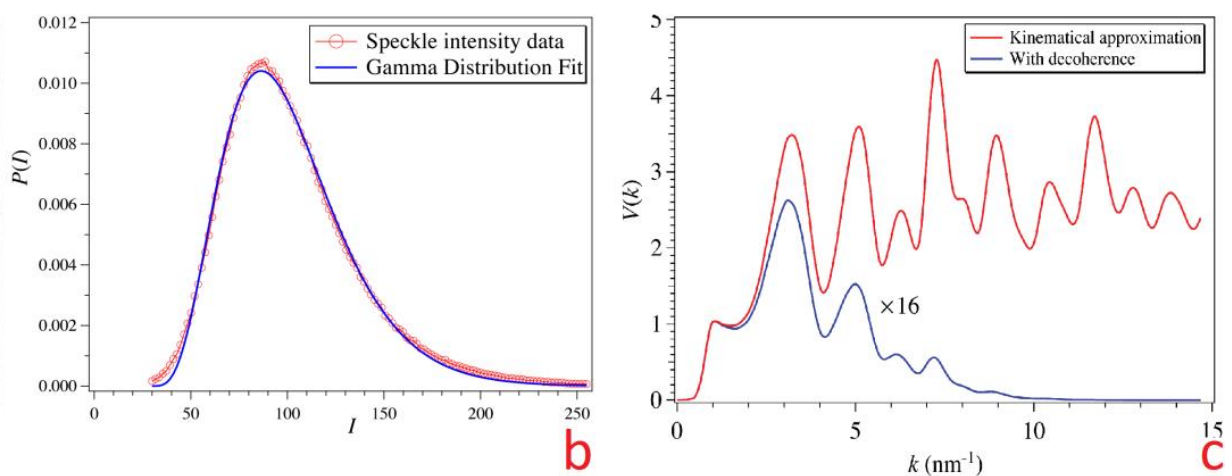

FIG 1. (a) Typical tilted dark-field image of a $20-\mathrm{nm}$ thick amorphous carbon illustrating the speckle distribution observed when the microscope resolution is $1.0 \mathrm{~nm}$ (Taken by a JEOL $2010 \mathrm{~F}$ at $200 \mathrm{kV}$, and a beam tilt of $\sim 4 \mathrm{~nm}^{-1}$ ). (b) The intensity histogram of this image, and the fit to a gamma distribution with $m \approx 30, I \approx 100$. (c) Normalize variance versus scattering vector computed for polycrystalline Si, 4 $\mathrm{nm}$ thick, and mean grain size of $1.2 \mathrm{~nm}$. The red plot is the coherent kinematic case. The blue plot is the output of the knock-on damage and multiple scattering simulations, giving an $m$ value nearer 16 in this instance. 